\title{
Measurement of Thermal Conductivity of Fibrous Insulation Using Guarded Hot Plate Method and Cyclic Heat Method
}

\author{
Takahiro OMURA \\ RD Dept. Hamamatsu Research Centre, NICHIAS Corporation. \\ 1-8-1 Shinmiyakoda, Hamamatsu, Shizuoka, 431-2103, Japan \\ E-mail :oomura-t@nichias.co.jp
}

\begin{abstract}
A thermal conductivity measuring apparatus using both the Guarded Hot Plate (GHP) method and the Cyclic Heat method was designed, and thermal conductivity of a fibrous insulations were measured in atmospheric conditions and in evacuated conditions at temperature range from 100 to $1300^{\circ} \mathrm{C}$. When the apparatus using the GHP method was designed, the sizes of the meter and guard heater are determined using the theory by William Woodside. In the Cyclic heat method apparatus, the error magnitude due to difference in period of heat wave was examined, and the time lag method was compared with the amplitude decay method.

The thermal conductivities of the fibrous insulations (aluminum-silica) of both the GHP method and the Cyclic Heat method are in good agreement. This result indicates that values of measurements by the nonsteady state method (Cyclic heat method) is in agreement with the steady state method (GHP method) on proper conditions.

As applied measurement, I compared thermal conductivity of the brick with the fibrous insulation in atmospheric conditions at temperature range from 100 to $1300^{\circ} \mathrm{C}$, and examined the relationship between heat flow and different types of fibrous fleeces in a fibrous insulation in evacuated conditions.
\end{abstract}

Key Word : Guarded Hot Plate (GHP) method, Cyclic Heat Method, thermal conductivity, fibrous insulation, brick, thermocouple, specific heat, Rockwool

\section{Introduction}

Thermal insulations, especially fibrous insulations, are widely used in many industries, including the space industry, the electric power industry. Recently, as it has been requested to use these in high temperature and evacuated conditions, it has been important to know their thermal conductivity in such conditions. The GHP method, which is one of the steady state methods, is generally used for measuring the thermal conductivity of thermal insulation. However there are few of reports about the thermal conductivity of thermal insulations in high temperature (more than $1000^{\circ} \mathrm{C}$ ) and evacuated conditions, because it is difficult for this method to measure in such conditions. Although non-steady state methods such as the Cyclic Heat method are applicable to measure the thermal conductivity in such conditions, there are few reports of fibrous insulation by these methods.

Therefore I have designed a thermal conductivity measuring apparatus using both the GHP method and the Cyclic Heat method, and have measured in atmospheric conditions and in evacuated conditions at temperature range from 100 to $1300^{\circ} \mathrm{C}$.

\section{Experiments}

\subsection{Principle}

The GHP method is a way of measuring thermal conductivity assuming one-directional heat flow. Thermal conductivity $\lambda$ can be expressed by following equation ${ }^{1,2)}$.

$$
\lambda=\frac{\mathrm{Q} \cdot \mathrm{d}}{\theta_{1}-\theta_{2}}
$$

Where $\lambda$ is thermal conductivity, $\mathrm{Q}$ is a heat flow, $\mathrm{d}$ is a specimen thickness, $\theta_{1}$ is the temperature of hot surface, and $\theta_{2}$ is the temperature of cold surface.

The Cyclic Heat method consists of two kinds of determining ways. One method is to measure the time lag of the phase of heat waves conducted through the specimen; the other is to measure the amplitude decay of a heat wave. Using either method we can determine thermal conductivity of the specimen by multiplying thermal diffusivity by specific heat and bulk density.

The time lag $\phi$ and the amplitude decay $A$ of a heat wave can be expressed by these equations ${ }^{3,4,5)}$.

$$
\begin{aligned}
& \phi=\arg \left\{\frac{\sinh k x(1+i)}{\sinh k L(1+i)}\right\} \quad \text { (2) } \quad A=\frac{A_{j}}{A_{s}}=\sqrt{\frac{\cosh 2 k x-\cos 2 k x}{\cosh 2 k L-\cos 2 k L}} \\
& k=\sqrt{\frac{\omega}{2 \kappa}}
\end{aligned}
$$

Where $\lambda$ is thermal conductivity, $\kappa$ is thermal diffusivity, $c$ is specific heat, $\rho$ is bulk density, $\phi$ is a time lag, $T$ is a period, $A$ 
is an amplitude decay, $L$ is a specimen's thickness, $x$ is the point of measuring temperature in the specimen and $k$ is defined by equation (4).

We can know the thermal conductivity of specimen by measuring $\phi$ and $A$, and using equations (2) (6).

\subsection{Apparatus}

\subsubsection{GHP method apparatus}

Fig 1 illustrates the new measuring apparatus of the GHP meth$\mathrm{od}^{1)}$. The apparatus consists of 4-parts, measuring part, controlling temperature part, analysis part (computer) and a vacuum pump.

For the single-sided mode of operation, there are one specimen, one cold plate (cold heater), and guarded hot plates (meter heater and guard heater) in measuring part. There is a gap between the meter and guard plates, and the gap width is $3 \mathrm{~mm}$. The meter and guard heater consist of 5-aluminum oxide plates, and the meter heater $(50 \times 50 \mathrm{~mm})$ is surrounded by 4-guard heater $(85 \times 32$ $\mathrm{mm})$. The other heaters, the cooling heater and the auxiliary heater, are $120 \mathrm{~mm}$ square. Each plate thickness is $3 \mathrm{~mm}$. An inner diameter of a cylindrical heater is $400 \mathrm{~mm}$. The thermal sensors are R-type thermocouples and the line-heat sources are Platinum wire $(\phi 0.6 \mathrm{~mm})$. There are 5-controllers that control each heater's temperature (the meter heater, the guard heater, the cold heater, the auxiliary heater and the cylindrical heater) in controlling temperature part.

Fig 2 shows the calculated errors $E_{e}$. The error $E_{e}$ is the ratio of

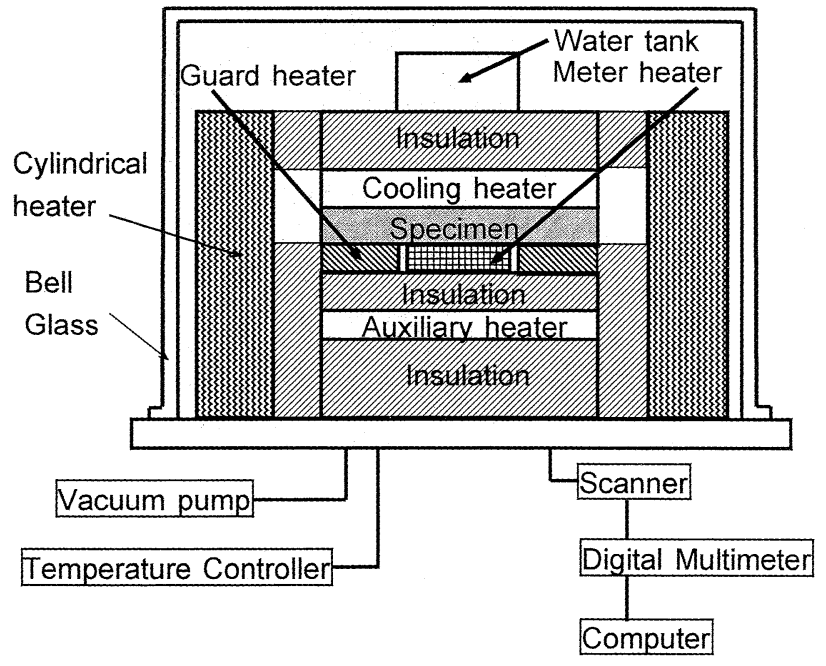

Figure 1 GHP method apparatus

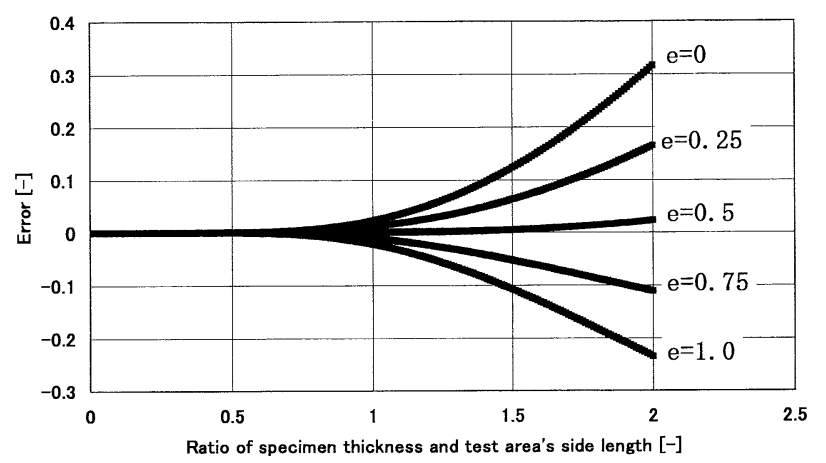

Figure 2 Calculated Errors heat flow $\phi_{\mathrm{e}}$ from edge surface to heat flow $\phi$ through specimen without heat flow from edge surface. And $e$ is given by following equation ${ }^{6,7)}$

$$
e=\frac{\theta-\theta_{2}}{\theta_{1}-\theta_{2}}
$$

Where $\theta_{1}$ is the bottom surface temperature, $\theta_{2}$ is the top surface temperature, and $\theta$ is the edge surface temperature.

$\mathrm{E}_{\mathrm{e}}$ is given by following equation ${ }^{6,7}$.

$$
\begin{aligned}
E_{e}=\frac{\phi_{\mathrm{e}}}{\phi}= & {\left[\frac { d } { \pi M } \left\{e \cdot \ln \frac{\cosh (\pi(b+M) / d)+1}{\cosh (\pi b / d)+1}\right.\right.} \\
& \left.\left.+(1-e) \ln \frac{\cosh (\pi(b+M) / d)-1}{\cosh (\pi b / d)-1}\right\}\right]^{2}-1
\end{aligned}
$$

Where $b$ is the guard heater's width, $M$ is half the length of a side of the meter heater, and $d$ is the specimen thickness.

$\phi$ is given by following equation.

$$
\phi=\frac{\lambda A \Delta \theta}{d}
$$

Where $\mathrm{A}$ is the heating surface area, $\lambda$ is the thermal conductivity of the specimen, and $\Delta \theta$ is the temperature difference.

This figure shows that the ratio of specimen thickness and test area's side length $(b+M)$ should be less than 1 for measuring thermal conductivity accurately. So the specimen's dimensions are determined $120 \mathrm{~mm}$ square and its thickness is less than $20 \mathrm{~mm}$, and the dimensions of the meter and guard heater are determined with these calculated errors.

Fig 3 shows an electric resistance of the meter heater as a function of absolute temperature. I use this function to obtain the power of the meter heater using Ohm's low, whenever measuring thermal conductivity using the GHP method.

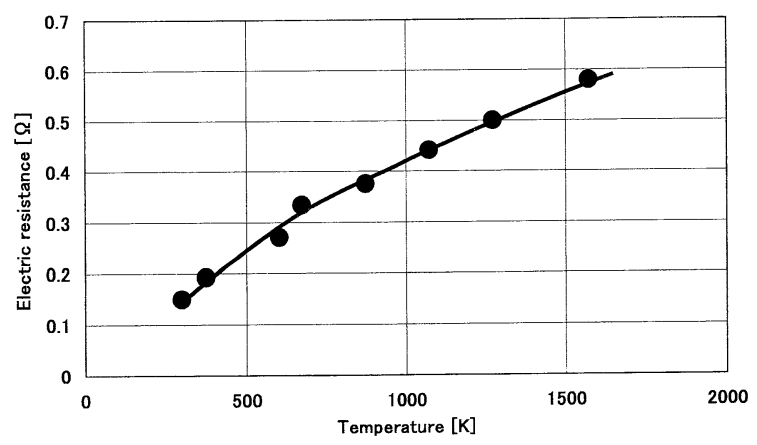

Figure 3 Electric resistance of the meter heater as a function of absolute temperature

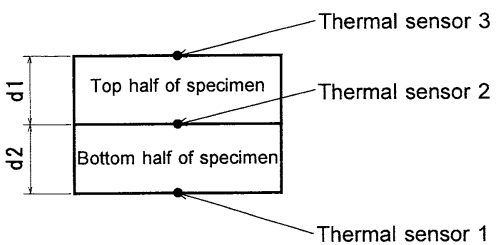

Figure 4 Specimen cross section 


\subsubsection{Cyclic Heat method apparatus}

When measuring thermal conductivity using the Cyclic Heat method, I have to change from the GHP method apparatus to the Cyclic Heat method apparatus. The cyclic heater is made by connecting the meter heater with the guard heater in series. Depending on the temperature selected for the test, the amplitude of the heat wave is from $2 \sim 66^{\circ} \mathrm{C}$ and the period is $1 \sim 2$ hours ${ }^{5)}$. Temperature of the top surface is kept constant. The measured temperature is the difference between the average values of the bottom surface's mean temperature and the top surface's mean temperature.

Fig 4 illustrates the cross-section of a specimen at measuring. The specimen consists of two halves, a top half and a bottom half. Thermal sensors are placed at three points. The thermal sensors are R-type thermocouples. A heat wave is generated at the bottom surface and it conducts through the specimen to the top surface. Specimen's dimensions of a half are $120 \mathrm{~mm}$ square and its thickness is $10 \sim 30 \mathrm{~mm}$.

One procedure measures the time lag of the phase of heat waves between the thermal sensor 1 and the thermal sensor 2 , and is called the time lag method. The other procedure measures the amplitude decay of the heat wave between the thermal sensor 1 and the thermal sensor 2, and is called the amplitude decay method. Using either method we can determine thermal conductivity of the specimen by multiplying thermal diffusivity by specific heat and density.

I examined how much error magnitude to a period of heat wave

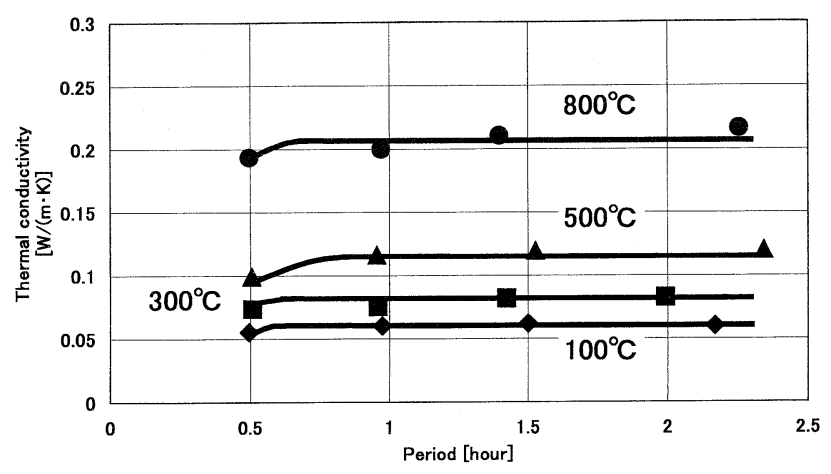

Figure 5 Thermal conductivity as a function of a period of heat wave

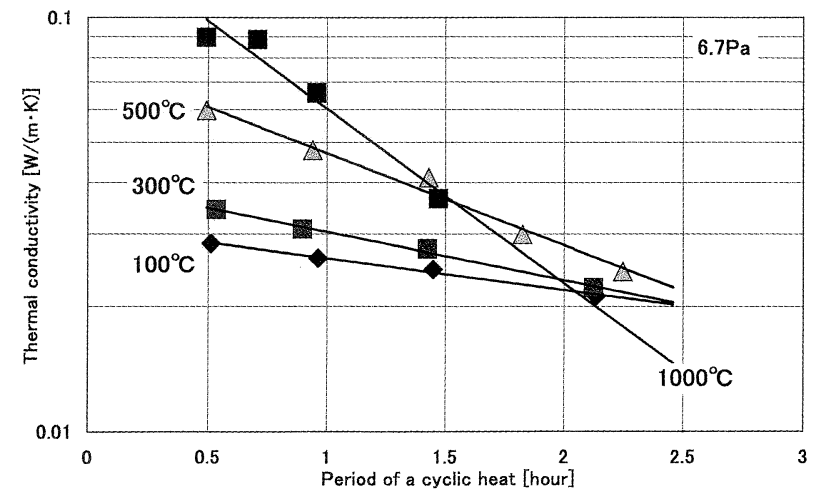

Figure 6 Thermal conductivity as a function of a period of a cyclic heat (Amplitude decay method) the thermal conductivity measuring apparatus has in the case of the Cyclic Heat method. Fig 5 shows thermal conductivity as a function of a period of the heat wave by using the time lag method. The specimen was a fibrous insulation (aluminum-silica, bulk density $; 130 \mathrm{~kg} / \mathrm{m}^{3}$ ) that had a bulk density of $130 \mathrm{~kg} / \mathrm{m}^{3}$. In this case I measured the thermal conductivity at $100^{\circ} \mathrm{C}, 300^{\circ} \mathrm{C}, 500^{\circ} \mathrm{C}$, and $800^{\circ} \mathrm{C}$. Thermal conductivity is nearly constant when the period of heat wave is longer than 1 hour.

In the case of the amplitude decay method these results contain much error, because of having heat loss from specimen's edge surface. Fig 6 shows thermal conductivity of a fibrous insulation (aluminum-silica, bulk density; $130 \mathrm{~kg} / \mathrm{m}^{3}$ ) as a function of a period of the heat wave by using the amplitude decay method. These result show that the thermal conductivities have the exponentially reduction of the heat waves ${ }^{5}$.

So I show thermal conductivities of fibrous insulations by using the time lag method in this paper.

\subsubsection{Specific heat measuring apparatus}

When calculating the thermal conductivity using the Cyclic Heat method, I have to know the specific heat of specimen.

Fig 7 shows the specific heat measuring apparatus. The apparatus is based on the comparison of the specific heat of water with the specimen's. A specimen that is heated in the furnace is dropped into the water tank 1 filled with water. The temperature of the water and the specimen are measured before being dropped and

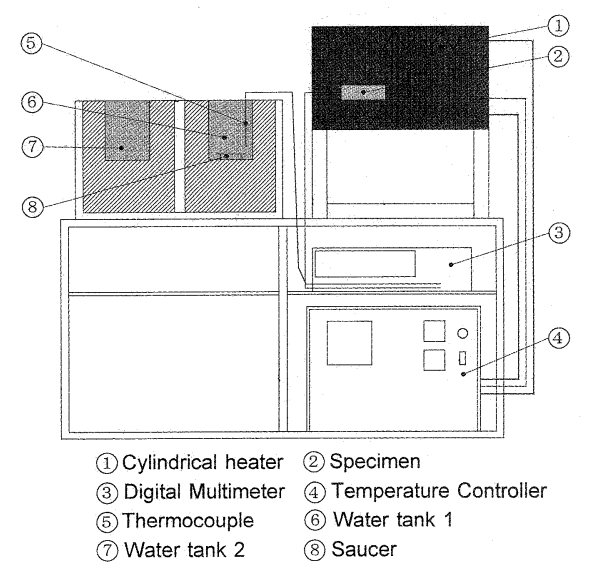

Figure 7 Specific heat measuring apparatus

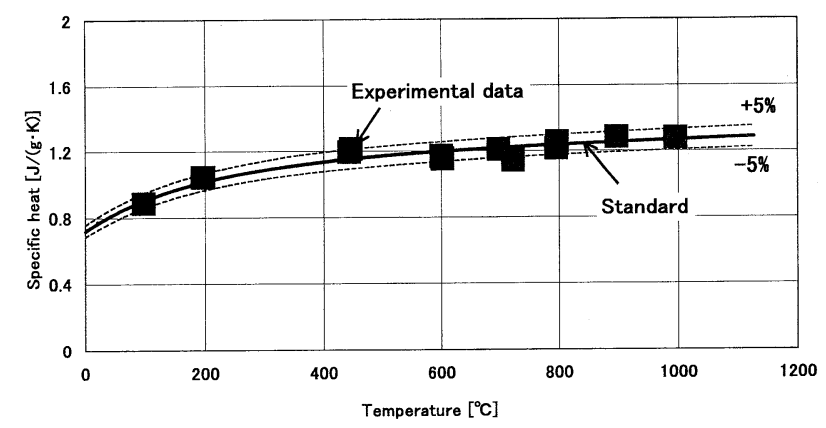

Figure 8 Specific heat of Synthetic Sapphire 
after being dropped. The specific heat of the specimen is obtained using the temperature difference between the water and the specimen before being dropped and after being dropped ${ }^{5)}$. In this measurement, the amount of evaporation from the water tank 1 is very important. It is derived by knowing the amount of evaporation from the water tank 2. The weight difference of 2-tank's water before being dropped and after being dropped is equal to the amount of evaporation.

I checked how much error magnitude this specific heat measuring apparatus has by measuring the specific heat of standard reference material. Fig 8 shows the specific heat of standard reference material (Synthetic Sapphire) that is obtained from the National Institute of Standards and Technology. Comparison of the measured values with the reference data indicates very good agreement ${ }^{5}$.

\section{Results and Discussion}

\subsection{Error magnitude to a period of heat wave}

Fig 5 shows that the value of the thermal conductivity of the specimen is small under a period of 0.5 hours or less. Two causes are thought.

One cause is that heat waves, which are conducted through a specimen, distort by a short period. When measuring the thermal conductivity of a thermal insulation under a short period, the heat waves are not sin-waves, because the thermal conductivity is very low.

The other is heat from the heat sink surface of the specimen where the thermal sensor 3 is mounted (Fig 4). This apparatus has an adequately adiabatic structure between top half of specimen and the water tank to measure thermal diffusivity in the temperature range from $100^{\circ} \mathrm{C}$ to $1300^{\circ} \mathrm{C}$. So heat flow through the specimen is limited, and heat waves are distorted at the period of about 0.5 hour.

\subsection{Comparison of the GHP method with the Cyclic Heat method}

Fig 9 shows the thermal conductivities of the fibrous insulation (aluminum-silica, bulk density : $205 \mathrm{~kg} / \mathrm{m}^{3}$ ) measured by the GHP method and the Cyclic Heat method as a function of temperature in atmospheric pressure and evacuated (the air pressure of $1.3 \mathrm{~Pa}$ ) conditions at temperature range from 100 to $1300^{\circ} \mathrm{C}$. Comparison of measured values using the GHP method with the Cyclic Heat method indicates very good agreement. Values of measurements

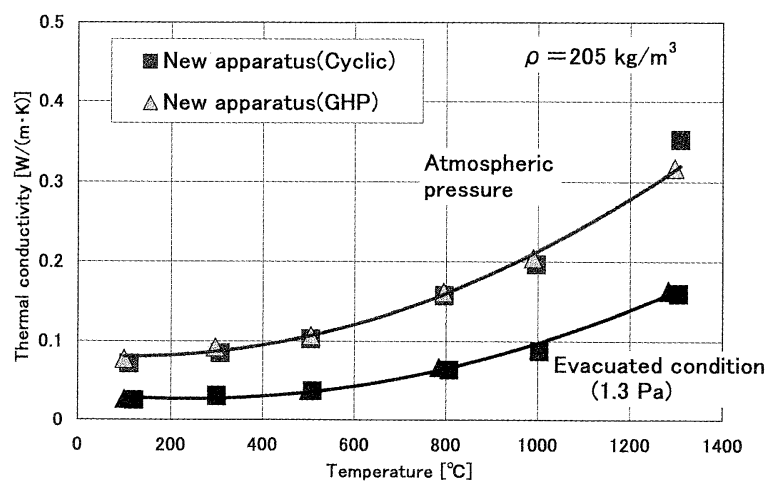

Figure 9 Thermal conductivity of the fibrous insulation as a function of temperature by the non-steady state method (Cyclic heat method) is not generally in agreement with the steady state method (GHP method), because in the non-steady state method, the thermal equilibrium between specimen surfaces and the circumference of the specimen is destroyed, natural convection generates in the specimen, and there are errors of responsiveness for measuring temperature, $\mathrm{etc}^{8}$. However, this result indicates that values of measurements by the non-steady state method (Cyclic heat method) is in agreement with the steady state method (GHP method) on proper conditions, which it is thought that the period of the heat waves is very important in the case of this measurement. Because the period is more than one hour in the cyclic heat method, it is thought that the thermal equilibrium in the specimen and between specimen surfaces and the circumference of the specimen is a condition comparatively similar to the steady state in a short time.

\subsection{Thermal conductivity of Fibrous insulation and Brick}

Fig 10 shows the thermal conductivities of the fibrous insulation (aluminum-silica, bulk density: $205 \mathrm{~kg} / \mathrm{m}^{3}$ ) and the brick (bulk density : $513 \mathrm{~kg} / \mathrm{m}^{3}$ ) by the GHP method as a function of the third power of the absolute temperature in the air pressure of $1.3 \mathrm{~Pa}^{1}$.

Heat transfer of a thermal insulation under evacuated condition is obtained by summation of heat transfer in solid and thermal radiation in a material. Therefore, effective thermal conductivity $\lambda$ of the thermal insulation under evacuated condition is equal to the summation of thermal conductivity $\lambda_{s}$ of solid and thermal conductivity $\lambda_{\mathrm{r}}$ of thermal radiation.

$$
\lambda=\lambda_{s}+\lambda_{r}
$$

$\lambda_{\mathrm{r}}$ is in proportion to the third power of the absolute temperature because $\lambda_{\mathrm{r}}$ is obtained from thermal radiation ${ }^{9)}$. Hence

$$
\lambda=\lambda_{s}+C T^{3}
$$

where $\mathrm{C}$ is constant. Therefore, if the $\mathrm{Y}$-axis is thermal conductivity $\lambda$ and the $\mathrm{X}$-axis is the third power of the absolute temperature, the intersection of equation (11) and the $\mathrm{Y}$-axis gives $\lambda_{\mathrm{s}}$ and the slope gives $\mathrm{C}$, a part of the effective thermal conductivity of thermal radiation. So we can know $\lambda_{\mathrm{s}}$ and $\lambda_{\mathrm{r}}$.

The difference in thermal conductivity between two materials is obvious throughout all absolute temperature ranges. However the slopes of both the functions almost agree. This result indicates that the difference in thermal conductivity between both materials is the cause of heat transfer in the solid. It is thought that the differences in the bulk density and structure (particle or fibrous) between two materials are the cause of the difference in thermal conductivity.

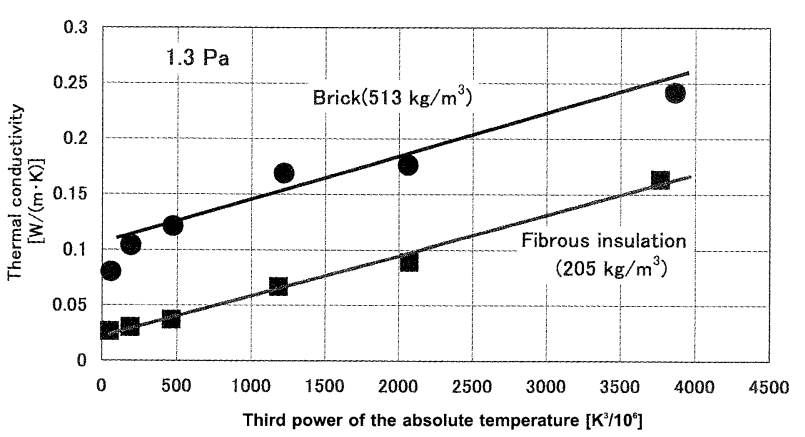

Figure 10 Thermal conductivity of Fibrous insulation and Brick as a function of the third power of the absolute temperature 
We find that the thermal conductivity of the brick does not vary directly at the lower temperature in Fig 10. The reason is that the ratio of the radiant heat transfer to the overall heat transfer decreases as temperature decreases, and heat transfer in the solid dominates. I believe that, compared to fibrous insulation, the brick's solid conductivity has strong influence on the thermal conductivity of the brick at low temperature. This is because the bulk density of the brick is greater than that of the fibrous insulation.

\subsection{Thermal conductivity of Rockwool insulation}

I examined the relationship between heat flow and different types of fibrous fleeces in a fibrous insulation (Rockwool insulation). The fibrous fleeces are like fibrous sheets. Fig 11 illustrates the two types of Rockwool insulations (bulk density: $240 \mathrm{~kg}$ / $\left.\mathrm{m}^{3}\right)^{4}$. One type was made of piling fiber fleeces perpendicular to heat flow (so called the perpendicular type), and the other was made of piling fiber fleeces parallel to heat flow (so called the parallel type).

Fig 12 shows thermal conductivity of two types of the Rockwool insulation as a function of the third power of the absolute temperature in evacuated conditions. I measured using the Cyclic Heat method. We find that the thermal conductivity of the perpendicular type is lower than that of the parallel type. Although the slopes of both the functions is slightly different, the difference of both the intersections of the line and the Y-axis is very large. Therefore, the difference of both results is the cause of thermal conductivity $\lambda_{s}$ of solid, and it is thought because thermal resistance of contact between the fibrous fleeces exists in the perpendicular type. By the way, the effective thermal conductivity of the radiation of the Parallel type being slightly greater than the perpen-

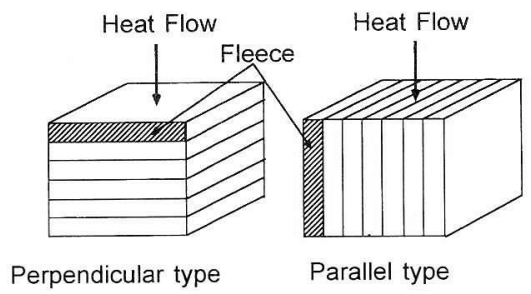

Figure 11 Schematic of the Rockwool insulation

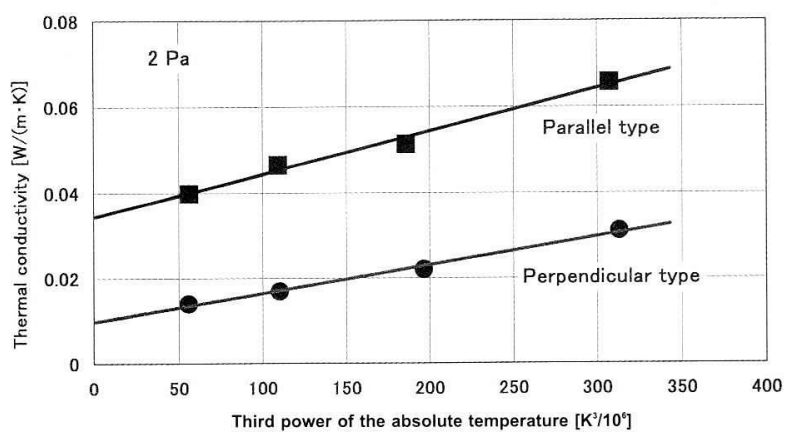

Figure 12 Thermal conductivity of Rockwool as a function of the third power of the absolute temperature dicular type is because the penetration of radiation is prevented by the fleeces perpendicular to the heat flow in the perpendicular type.

\subsection{Corrosion of thermocouples}

If thermal sensors, which I used as R-type thermocouples, are exposed under high temperature and evacuated conditions for a long time, they will corrode. The corrosion of the thermocouples will especially cause error in the measured value using the GHP method.

I had measured the thermal conductivity under high temperature (more than $1000^{\circ} \mathrm{C}$ ) and evacuated conditions for half a year, and found that the measured temperatures at each thermocouple decreased about $10 \sim 25^{\circ} \mathrm{C}$ over this time period. Fig 13 and Fig 14, which are photographs taken using the scanning electron microscope, show the cross section of the cut surface of thermocouples. Fig 13 shows a thermocouple before being used. We can see that the cut surface is clean. Fig 14 shows a thermocouple after being used for about 100 hours under the temperature of $1300^{\circ} \mathrm{C}$.

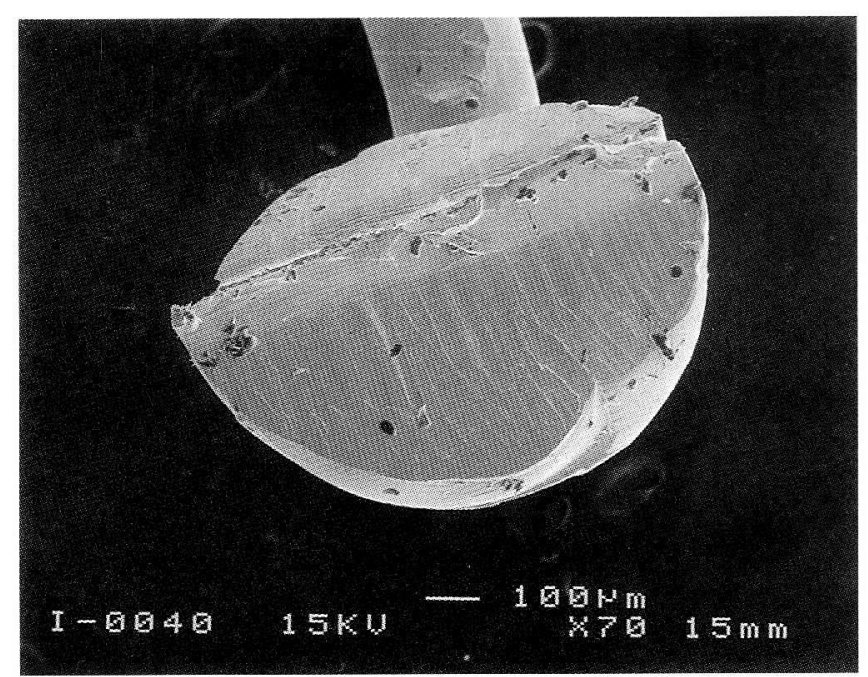

Figure 13 SEM images of the cross section of the cut surface of thermocouple before being used

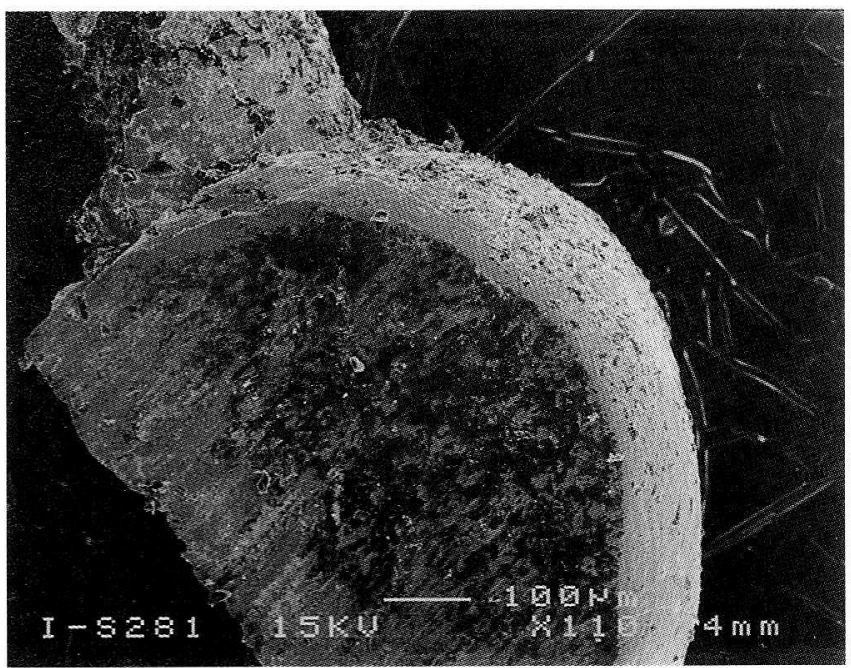

Figure 14 SEM images of the cross section of the cut surface of thermocouple after being used 
We can see that the cut surface is not clean and is corroded. Analysis indicated that aluminum oxide and silicon dioxide ( $\mathrm{Si}$ $\mathrm{O}_{2}$ ) were detected in the dark parts in Fig 14. These materials are components of the heaters and specimens. I believe that the thermocouples were corroded by the penetration of aluminum oxide and silicon dioxide $\left(\mathrm{SiO}_{2}\right)$.

This result indicates that the thermocouples have a limited lifetime of measuring under high temperature and evacuated conditions. I have to change thermocouples at regular interval that depends on the measuring conditions (temperature, air pressure).

\section{Conclusion}

A thermal conductivity measuring apparatus using both the GHP method and the Cyclic Heat method is designed. Comparison of measured values using the GHP method with the Cyclic Heat method indicates very good agreement under atmospheric and evacuated conditions at temperature range from 100 to $1300^{\circ} \mathrm{C}$. So this result indicates that values of measurements by the non-steady state method is in agreement with the steady state method on proper conditions

I compared thermal conductivity of the brick with the fibrous insulation. Thermal conductivity of the brick is greater than the fibrous insulation. The difference in thermal conductivity $\lambda_{s}$ of solid is the reason for the difference in thermal conductivity of materials.

I examined the relationship between heat flow and different types of fibrous fleeces in a fibrous insulation. The fleeces perpendicular to the heat flow are significant for preventing heat conduc- tion.

I examined the corrosion of thermocouples exposed under high temperature and evacuated conditions for a long time. Thermocouples were corroded by the penetration of aluminum oxide and silicon dioxide $\left(\mathrm{SiO}_{2}\right)$. The thermocouples have a limited lifetime of measuring under high temperature and evacuated conditions.

\section{References}

1) T.Omura, Y,Kawasaki ; 18th Japan Symposiumon Thermophysical Properties B222, (1997)

2) ASTM. C177, "Standard Test Method for Steady-State Heat Flux Measurements and Thermal Transmission Properties by Means of the Guarded-Hot-Plate Apparatus", Annual Book of ASTM Standard, Vol 04.06

3) H.S.Carslaw, J.C.Jaeger ; Conduction of Heat in Solids, p.105, (OXFORD, Second edition, 1959)

4) T.Omura, M,Nakayama; 18th Japan Symposiumon Thermophysical Properties B221, (1997)

5) T.Omura, M.Tsuboi ; Netsu Busei 13 [4], pp.264-270, (1999)

6) Handbook of Japanese Industrial Standard, pp.372-374, (Japanese Thermal Insulation Association, 1995)

7) William Woodside; "ANALYSIS OF ERRORS DUE TO EDGE HEAT LOSS IN GUARDED HOT PLATE", ASTM STP, pp.49-64, (1957),

8) Netsu Busseiti Sokuteihou, pp,4-5, (YOKENDO, 1991)

9) Stanley D.Williams, Donald M. Curry ; "Prediction of Rigid Silica Based Insulation Conductivity", NASA Technical Paper 3276 (1993). 